\title{
Statistics of multimoding in optimum compensation of reactive loads of electrical systems
}

\author{
Alexey Gerasimenko ${ }^{1, *}$ \\ ${ }^{1}$ Siberian Federal University, Krasnoyarsk, Russia
}

\begin{abstract}
A stochastic approach has been implemented to account for multimodality to optimize the operating conditions of electrical systems. On the basis of the algorithm for optimization of the instantaneous mode and the stochastic model of the load graphs, a mathematical model of the generalized reduced gradient was obtained. The practical implementation of the algorithm was carried out due to its low labor intensity.
\end{abstract}

Keywords. statistical model, optimal reactive load compensation, generalized reduced gradient method, electrical systems,

\section{Introduction}

Optimal reactive load compensation (RLC) in the grids of electrical systems (ES) can significantly increase the efficiency of their functioning. The problem solving here requires taking into account the aggregate of steady-state modes (multimoding), obtaining and analyzing the integral parameters of this aggregate, such as energy loss in the system, ranges and diagrams of voltages and reactive power changes in its nodes. Direct optimization of each mode separately, generalization and analysis of economic and mode characteristics of the aggregate of modes dramatically complicates the problem at hand, making it excessively cumbersome and time-consuming. Against this background, the most productive is the gradient methods, proved themselves in operational problems, using the probabilistic-statistical (stochastic) approach, which allows solving problems more strictly than deterministic approaches [1]. A statistical model for accounting the set of steady-state modes [2,3] combined with a generalized reduced gradient (GRG) method $[4,5]$ are the basis of the developed optimization method. The basic principles for solving the problem of optimal RLC in terms of the minimum loss of electric energy (EE) is given below.

\section{Statistical Model of Electrical Loads}

The optimal RLC is formulated as a nonlinear mathematical programming problem [4]; it belongs to the stochastic optimization since loads of the ES nodes are random variables. The stochastic approach of taking into account multimoding [2, 3], caused by the change in electric loads, is implemented based on an analytical model of load changes which is obtained through factor (component) analysis [6, 7]. Electrical loads

\footnotetext{
* Corresponding author: gerasimenkoaa@yandex.ru
}

modelling on the basis of factor analysis allows identifying common and most stable patterns of changing the configuration of load curves, compressing the information regarding the multimoding through a small number of generalizing factors with their subsequent application of them when calculating the EE losses and other integral parameters.

The load diagrams modelling using the principal component analysis is as follows. Based on a representative sample $N$ of initial load diagrams, we determine a moment correlation matrix (MCM) and select $M$ of maximum eigenvalues $\lambda_{i}$ and their corresponding eigenvectors $\bar{v}_{i}$. These values determine the main factors - generalized (orthogonal) load diagrams (GLD):

$\mathrm{G}_{k j}=\sum_{i=1}^{N} v_{k i}^{\prime} \Delta P_{i j}+\sum_{i=1}^{N} v_{k i}^{\prime \prime} \Delta Q_{i j} j=\overline{1, d}, k=\overline{1, M}$,

where: $v_{k i}^{\prime}, v_{k i}^{\prime \prime}$ are the components of eigenvector $\bar{v}_{k}$ of MCM; $\Delta P_{i j}, \Delta Q_{i j}$ are components $j$ of the centered diagrams of active $P_{i}$ and reactive $Q_{i}$ of the loads of node $i$ with $d$ intervals of constancy.

The modelling GLDs are a set of statistically independent basis vectors oriented so that each of them reflects most of the connection of the initial aggregate of load diagrams and contributes most to the variance of the initial variables. Like eigenvectors, GLDs are orthogonal (statistically independent), uncorrelated (unrelated) quantities. They attribute the properties of linearity and additivity to the models, the statistical method, and the whole process of multimoding modelling. Analysis of the GLD configurations for various realizations of the random 
process of changing the loads showed the presence of statistical stability of these factor models, i.e. proximity of the numerical characteristics of the corresponding GLDs of various samples of the original diagrams.

This statistical transformation of MCM helps simulate fairly accurately the original curves of electrical loads $P_{i j}$ and $Q_{i j}$ using known mathematical expectations $M P_{i}$ and $M Q_{i}$ and simulated deviations of loads from mathematical expectations in the form of M-linear combinations of statistically stable GLD:

$$
\begin{gathered}
P_{i j}=M P_{i}+\sum_{k=1}^{M} v_{k i}^{\prime} \mathrm{G}_{k j} ; \quad Q_{i j}=M Q_{i}+\sum_{k=1}^{M} v_{k i}^{\prime \prime} \mathrm{G}_{k j}, \\
i=\overline{1, N}, \quad j=\overline{1, d} .
\end{gathered}
$$

Such a representation of the loads turned out to be effective since obtaining model (2) of acceptable accuracy needs only to take into account up to the first three or four GLDs $(M<<N)$, which reflect up to $85-95 \%$ of the total variance of the initial loads. The modelling error for the interval values of unknown load curves by models (2), being in the range of $\pm(2-15) \%$, is not a crucial factor for determining the integral characteristics since its influence decreases as a result of stepwise (iterative) refinement of pseudo-average loads and, accordingly, load diagrams models in the combined algorithm for determining these parameters.

The solution for optimal RLC is based on taking into account the entire set of modes in the form of their integral characteristics, primarily EE losses, which are a target criterion for solving the operational problem with determining the optimal load of existing reactive power sources.

\section{Statistical modelling of the set of steady-state modes [2, 3]}

The general expression of the EE load losses in the ES with $m$-branches is basically determined by accurate summing (integrating) of power losses $\Delta P$ at all time intervals $\Delta t$ (in all modes) of calculated period $T$ according to the classical expressions:

$$
\begin{aligned}
& \Delta \mathrm{E}_{11}=3 \sum_{j=1}^{m} R_{j} \int_{0}^{T} I_{j}^{2}(t) d t= \\
& \sum_{j=1}^{m} \int_{0}^{T} \Delta \mathrm{P}_{j}(t) d t=\sum_{i, j}^{N+1} \int_{0}^{T} \Delta P_{i j}(t) d t \approx \\
& \sum_{i, j}^{N+1} \sum_{k=1}^{d} \Delta P_{i j k} \Delta t_{k} .
\end{aligned}
$$

The EE load losses are the sum of the main component $\mathrm{M} \boldsymbol{\Delta} \boldsymbol{E}$ determined for the average loads mode, and the variance component $\boldsymbol{\sigma} \boldsymbol{\Delta} \boldsymbol{E}$ which takes into account the deviation of the loads from the average values:

$$
\begin{aligned}
& \Delta E=M \Delta E+\sigma \Delta E=\Delta P(M \bar{V}, M \bar{\delta}) T+\left[\frac{1}{2} \sum_{i=1}^{N} \sum_{j=1}^{N} k\left(V_{i} V_{j}\right) \frac{\partial^{2} \Delta P}{\partial V_{i} \partial V_{j}}+\right. \\
& \left.+\sum_{i=1}^{N} \sum_{j=1}^{N} k\left(V_{i} \delta_{j}\right) \frac{\partial^{2} \Delta P}{\partial V_{i} \partial \delta_{j}}+\frac{1}{2} \sum_{i=1}^{N} \sum_{j=1}^{N} k\left(\delta_{i} \delta_{j}\right) \frac{\partial^{2} \Delta P}{\partial \delta_{i} \partial \delta_{j}}\right] T
\end{aligned}
$$

where $\Delta P(M \bar{V}, M \bar{\delta}), k\left(V_{i} V_{j}\right), k\left(V_{i} \delta_{j}\right), k\left(\delta_{i} \delta_{j}\right)$ are power losses, correlation moments calculated for moduli $M V$ and phases $M \delta$ of the voltages at the point corresponding to the mathematical expectations of the loads; $\frac{\partial^{2} \Delta P}{\partial V_{i} \partial V_{j}}, \frac{\partial^{2} \Delta P}{\partial V_{i} \partial \delta_{j}}, \frac{\partial^{2} \Delta P}{\partial \delta_{i} \partial \delta_{j}}-$ second derivatives of the expression of power losses with respect to the corresponding variables, they have been calculated relevantly to the same point; $N$ - the number of nods in the circuit without a slack bus.

The main component of EE losses is determined by calculating the steady-state mode (SSM) for average loads with high reliability. The greatest difficulty is a complete and simple calculation of the multimoding when calculating the variance component, which is a critical factor in the EE loss analysis in general.

The EE loss expression (4) is featured by the correlation moments of moduli $V$ and phases $\delta$ of the voltages, which form the MCM of the voltages, which are obtained on the basis of a system of equations written similarly to the linearized equations of nodal voltages (NVEs):

$$
\left[\begin{array}{c:c}
\partial P_{i} / \partial \delta_{j} & \partial P_{i} / \partial V_{j} \\
\hdashline \partial Q_{i} / \partial \delta_{j} & \partial \bar{Q}_{i} / \partial V_{j}
\end{array}\right]\left[\begin{array}{l}
\Delta \overline{\delta_{i}} \\
\Delta \overline{V_{i}}
\end{array}\right]=\left[\begin{array}{c}
\Delta \overline{P_{i}} \\
\Delta \overline{Q_{i}}
\end{array}\right], \quad i, j=\overline{1, N},
$$

where $\Delta P_{i}, \Delta Q_{i}, \Delta V_{i}, \Delta \delta_{i}-$ deviations of active, reactive powers, moduli, phases of nodal voltages from their mathematical expectations.

Since the deviations of the voltages and wattages from their mathematical expectations are approximately related by the NVE system (5), the centered random parameters (variations of the voltage phases and moduli) $\Delta \delta_{i}, \Delta V_{i}$, same as $\Delta P_{i}, \Delta Q_{i}$, similarly to (2) are formulated by linear combinations of GLD:

$$
V_{i j}=M V_{i}+\sum_{k=1}^{M} \gamma_{k i}^{\prime \prime} \mathrm{G}_{k j} \quad \delta_{i j}=M \delta_{i}+\sum_{k=1}^{M} \gamma_{k i}^{\prime} \mathrm{G}_{k j} i=\overline{1, N}, j=\overline{1, d} .
$$

After substituting deviation of the mode parameters from expressions (2) and (6) into the system (5), coefficients $\gamma_{k i}^{\prime}, \gamma_{k i}^{\prime \prime}$, modeling the deviations of the phases and voltage moduli from the average values are calculated from equations equivalent to the system of linearized NVEs:

$$
[J] \times\left[\begin{array}{l}
\bar{\gamma}_{k i}^{\prime} \\
{\overline{\gamma^{\prime \prime}}}_{k i}
\end{array}\right]=\left[\begin{array}{l}
{\overline{v^{\prime}}}_{k i} \\
{\overline{v^{\prime \prime}}}_{k i}
\end{array}\right], k=\overline{1, M}, i=\overline{1, N},
$$

where $v_{k i}^{\prime} \gamma_{k i}^{\prime}, \quad v_{k i}^{\prime}, v_{k i}^{\prime \prime}$ are determined with initial (reconstructed) load diagrams and GLD according to (2) as: $v_{k i}^{\prime}=\frac{1}{d} \sum_{j=1}^{d} \mathrm{G}_{k j} \Delta P_{i j} ; v_{k i}^{\prime \prime}=\frac{1}{d} \sum_{j=1}^{d} \mathrm{G}_{k j} \Delta Q_{i j}, k=\overline{1, M}, i=\overline{1, N}$. 
The stochastic model of load diagrams (1)-(2), (6)-(8) allows expressing the MCM elements of voltages and wattages with modeling coefficients

$$
\begin{array}{r}
k\left(\delta_{i} \delta_{j}\right)=\sum_{k=1}^{M} \gamma_{k i}^{\prime} \gamma_{k j}^{\prime} ; k\left(V_{i} \delta_{j}\right)=\sum_{k=1}^{M} \gamma_{k i}^{\prime \prime} \gamma_{k j}^{\prime} ; \\
k\left(V_{i} V_{j}\right)=\sum_{k=1}^{M} \gamma_{k i}^{\prime \prime} \gamma_{k j}^{\prime \prime} ; \sigma^{2} \delta_{i}=\sum_{k=1}^{M} \gamma_{k i}^{\prime 2} ; \sigma^{2} V_{i}=\sum_{k=1}^{M} \gamma_{k i}^{\prime \prime 2} .
\end{array}
$$

Similar to (9) for the elements, MCMs of wattages are:

$$
\begin{gathered}
k\left(P_{i} P_{j}\right)=\sum_{k=1}^{M} v_{k i}^{\prime} v_{k j}^{\prime}, k\left(P_{i} Q_{j}\right)=\sum_{k=1}^{M} v_{k i}^{\prime} v_{k j}^{\prime \prime}, k\left(Q_{i} Q_{j}\right)=\sum_{k=1}^{M} v_{k i}^{\prime \prime} v_{k j}^{\prime \prime}, \\
\sigma^{2}\left(P_{i}\right)=\sum_{k=1}^{M}\left(v_{k i}^{\prime}\right)^{2} ; \sigma^{2}\left(Q_{i}\right)=\sum_{k=1}^{M}\left(v_{k i}^{\prime \prime}\right)^{2}
\end{gathered}
$$

Considering the correlation moments for moduli and phases of voltages (9), the EE load losses (4) are:

$$
\begin{aligned}
& \Delta E=[\Delta P(M \bar{V}, M \bar{\delta})+\sigma \Delta P] T=\left[\Delta P(M \bar{V}, M \bar{\delta})+\frac{1}{2} \sum_{k=1}^{M} \sum_{i=1}^{N} \sum_{j=1}^{N} \gamma_{k \gamma}^{\prime \prime} \gamma_{k j}^{\prime \prime} \frac{\partial^{2} \Delta P}{\partial V_{i} \partial V_{j}}+\right. \\
& \left.+\sum_{k=1}^{M} \sum_{i=1}^{N} \sum_{j=1}^{N} \gamma_{k i}^{\prime \prime} \gamma_{k j}^{\prime} \frac{\partial^{2} \Delta P}{\partial V_{i} \partial \delta_{j}}+\frac{1}{2} \sum_{k=1}^{M} \sum_{i=1}^{N} \sum_{j=1}^{N} \gamma_{k i}^{\prime} \gamma_{k j}^{\prime} \frac{\partial^{2} \Delta P}{\partial \delta_{i} \partial \delta_{j}}\right] T \cdot(11)
\end{aligned}
$$

Analysis of the accuracy of calculating the EE losses by the statistical testing with 2-4 GLD made for samples of test circuits of power grids with $35,110,220 \mathrm{kV}$, a small number of nods (up to 10), and real-world 6-220 kV circuits of power grids within the Krasnoyarsk energy system with up to 25 nods, resulted in finding that these expressions allow to compute load losses with acceptable accuracy: considering significance level of 0.95 for the samples, the average error in calculating EE losses for test circuits was $\boldsymbol{\delta}_{\boldsymbol{a} v}=-(1,2 \div 1,7) \%$ with $\sigma 2$ scattering up to 0.70 , and $\boldsymbol{\delta}_{\boldsymbol{a} v} \pm(0,25 \div 0,45) \%$ with $\sigma^{2}$ scattering up to 0.64 for real-world circuits.

EE losses of idle running $N_{T}$ of transformers with $g_{i}^{T}$ conduction are specified in the initial and optimal modes in accordance with the obtained voltage diagrams (6):

$$
\Delta E_{\mathrm{X}}=\sum_{i=1}^{N_{T}} g_{i}^{T} \int_{0}^{T} V_{i}^{2}(t) d t \approx \sum_{i=1}^{N_{T}} \sum_{j=1}^{d} g_{i}^{T} V_{i j}^{2} t_{j} \approx T \sum_{i=1}^{N_{T}} g_{i}^{T} M V_{i}^{2} .
$$

Linear system of equations in SSM (5), factor transforming (1), load model (2) and relations (6) - (12), expressing deviations of dependent variables $(\delta, V)$ and their second moments through the corresponding characteristics of independent variables $(P, Q)$, collectively form a statistical model for the SSM analysis and ES multimolding analysis.

Finding the integral characteristics through the stochastic method (7) - (12) does not require interval calculations of the modes; it comes down to one calculation of the SSM of the electrical system for average loads and an additional solution of three to four systems of linear equations (7) with the implacable Jacobian matrix, which drastically simplifies calculations of multimoding and EE losses in general, in comparison with direct dcalculations of the SSM (3) over the intervals for averaging the electrical load diagrams. The method allows to calculate electric power losses and other integral characteristics with accuracy and reliability sufficient for practice. Moreover, in comparison with the deterministic methods, the tolerance to random errors increases. EE load losses can be calculated via any algorithm computing the SSM which can be supplemented by blocks for determining (7), (8) of the modulating coefficients $v_{k i}^{\prime}, v_{k i}^{\prime \prime}$ and $\gamma_{k i}^{\prime}, \gamma_{k i}^{\prime \prime}$, which usually make the analysis only, $20-40 \%$ more laborious.

\section{Mathematical model for stochastic optimization of modes}

The basis of the model is the multimode-based GRG constructing apparatus with the statistical accounting of multimoding [8, 9]. While solving the operational problem, we define the minimum of the objective function of the total EE losses (11), (12) under the balance nonlinear equality constraints (NVEs) for the mathematical expectation of mode parameters, and simple inequality constraints

$Q_{i}^{\min } \leq Q_{i} \leq Q_{i}^{\max }, i=\overline{1, G} ; V_{i}^{\min } \leq V_{i} \leq V_{i}^{\max }, i=\overline{1, N}$,

where $G$ is a number of nods with sources of reactive power (RP).

Epy constraints (13) all be applied to the entire time interval, i.e. for each mode, which should be controlled in two ways:

1) modelling with GLD for RP (2) and voltages (6) at each optimization step and checking compliance with the constraints (13);

2) calculating of design ranges for variation of the considered parameters, which, considering variances (9), (10), are determined by Chebyshev's inequalities:

$$
\begin{gathered}
V_{i}^{\min \mathrm{p} .}=M V_{i}-k_{\beta}^{\min } \sigma V_{i} ; V_{i}^{\max \mathrm{p} .}=M V_{i}+k_{\beta}^{\max } \sigma V_{i} ; \\
\left.\begin{array}{c}
Q_{i}^{\min \mathrm{p} .} \\
=M Q_{i}-k_{\beta}^{\min } \sigma Q_{i} ; Q_{i}^{\max \mathrm{p} .}=M Q_{i}+k_{\beta}^{\max } \sigma Q_{i} \cdot
\end{array}\right\} \\
V_{i}^{\max \mathrm{p} .} \leq V_{i}^{\max } ; V_{i}^{\min \mathrm{p} .} \geq V_{i}^{\min } \\
Q_{i}^{\max \mathrm{p} .} \leq Q_{i}^{\max } ; Q_{i}^{\min \mathrm{p} .} \geq Q_{i}^{\min }
\end{gathered}
$$

Considering antisymmetric (biased) function of distribution density of $V_{i}, Q_{i}$, the values of $k_{\beta}$, ensuring the minimum error of the interval analysis on average up to 5$10 \%$, is justified for the range: $k_{\beta}^{\min }=1,45-1,55$, $k_{\beta}^{\max }=1,55-1,65$ with a significance level of $\beta=$ 0,90 .

Dependent (basic) $\bar{X}$ and independent regulated) $\bar{Y}$ variables that make up the general vector are the key parameters for forming array expression for determining the reduced gradient, and the expectations of mode 
parameters and active constraints (13) are as follows:

$\left\{\begin{array}{l}\left(V_{1 i}, Q_{2 j}\right) \in \bar{Y} \rightarrow V_{1 i} \in V_{\mathrm{marg}}, Q_{2 j} \in Q_{\mathrm{perm}} ; i=\overline{\mathbf{1}, \boldsymbol{p}} ; j=\overline{\mathbf{1}, \boldsymbol{q}} ; \boldsymbol{p}+q=G ; i \neq j ; \\ \left(V_{2 i}, \delta_{j}, Q_{1 l}\right) \in \bar{X} \rightarrow i=\overline{\mathbf{1}, N-\boldsymbol{p}} ; j=\overline{\mathbf{1}, N} ; l=\overline{\mathbf{1 , p}}\end{array}\right\}$

where $\boldsymbol{V}_{\text {marg }}, \boldsymbol{Q}_{\text {perm }}$ are a set of marginal voltages and admitted values of RM sources respectively; 1 and 2 are the indices of dependent and independent variables; $p, q$ are the number of independent variables within $V, Q$.

If simple constraints (13) are violated, the basic set changes. This means exchanging corresponding components between vectors $\overline{\boldsymbol{X}}$ and $\overline{\boldsymbol{Y}}$.

The following separation of variables is proposed for the components of eigenvectors and modeling coefficients:

$$
\left\{\begin{array}{l}
\left(v_{k 2 i}^{\prime \prime}, \gamma_{k 1 j}^{\prime \prime}\right) \in \bar{Y} \rightarrow k=1 ; i=\overline{1, q} ; j=\overline{1, p} ; q+p=G ; i \neq j ; \\
\left(v_{k 1 i}^{\prime \prime}, \gamma_{k 2 j}^{\prime \prime}\right) \in \bar{X} \rightarrow k=1 ; i=\overline{1, p} ; j=\overline{1, N-p} ; i \neq j ; \\
v_{k 1 i}^{\prime \prime} \in \bar{Y} \rightarrow k=\overline{2, M} ; i=\overline{1, G} ; \\
\gamma_{k i}^{\prime \prime} \in \bar{X} \rightarrow k=\overline{2, M} ; i=\overline{1, N} ; \gamma_{k i}^{\prime} \in \bar{X} \rightarrow k=\overline{1, M} ; i=\overline{1, N} .
\end{array}\right\}
$$

In this case, a basic set change is provided only for the variables reflecting multimoding with the first GLD $(\mathrm{M}=1)$.

Using the matrixed linearized system (5), provided the mode is balanced for the active power $\left(\Delta P_{i}=0\right)$, following the illustrated separation of variables (16), with the subsequent grouping of the vectors of the dependent and independent variables, we obtain the system of equations reflecting the parameters of SSM corresponding to the loads expectations:

$$
\left[\begin{array}{c:c:c:c}
E & \overline{0} & \overline{0} & A_{12} \\
\hdashline \overline{0} & E & \overline{0} & A_{22} \\
\hdashline \overline{0} & \overline{0} & E & -B_{12} \\
\hdashline \overline{0} & \overline{0} & -B_{21}^{-1} & E
\end{array}\right]\left[\begin{array}{c} 
\\
\Delta \bar{\delta} \\
\hdashline \Delta \bar{Q}_{1} \\
\hdashline \Delta \bar{V}_{2}
\end{array}\right]+\left[\begin{array}{c:c}
A_{11} & \overline{0} \\
\hdashline A_{21} & \overline{0} \\
\hdashline-B_{11} & \overline{0} \\
\hdashline \overline{0} & -B_{22}^{-1}
\end{array}\right]\left[\begin{array}{c}
\Delta \bar{V}_{1} \\
\hdashline \Delta \bar{Q}_{2}
\end{array}\right]=\overline{0}
$$

When constraints are inactive in (13), equations (17) are reduced to equations with identity matrix

$$
\left[\begin{array}{c:c}
E & A_{22} \\
\hdashline \overline{0} & E
\end{array}\right]\left[\begin{array}{c}
\Delta \bar{\delta} \\
\hdashline \Delta \bar{V}
\end{array}\right]+\left[\begin{array}{c}
\overline{0} \\
\hdashline-B_{22}^{-1}
\end{array}\right][\Delta \bar{Q}]=\overline{0} .
$$

In systems (17), (18), the following matrices are used to relate the dependent and independent mode parameters

$$
\begin{aligned}
& A=\left[\begin{array}{c:c}
A_{11} & A_{12} \\
\hdashline A_{21} & A_{22}
\end{array}\right]=\left[\frac{\partial P}{\partial \delta}\right]^{-1}\left[\frac{\partial P}{\partial V}\right] ; \\
& B=\left[\begin{array}{c:c}
B_{11} & B_{12} \\
\hdashline B_{21} & B_{22}
\end{array}\right]=\left[\frac{\partial Q}{\partial V}\right]-\left[\frac{\partial Q}{\partial \delta}\right] A .
\end{aligned}
$$

Following the separation of variables (16), on the basis of expressions (2) and (5) - (7), considering pairwise equivalence of variables $\gamma^{\prime}$ and $\delta, \gamma^{\prime \prime}$ and $V, U^{\prime \prime}$ and $Q$, equality constraints (NVEs) taking into account multimolding are the most completely simulated by the system of equations (17) representing mathematical expectations of the parameters being optimized, and the following systems of equations considering deviations of parameters from mathematical expectations:

$$
\left[\begin{array}{c:c:c:c}
E & \overline{0} & \overline{0} & A_{12} \\
\hdashline \overline{0} & E & \overline{0} & A_{22} \\
\hdashline 0 & 0 & E & -B_{12} \\
\hdashline \overline{0} & \overline{0} & -B_{21}^{-1} & E
\end{array}\right]\left[\begin{array}{c}
\overline{\gamma^{\prime}} \\
\hdashline \overline{v_{1}^{\prime \prime}} \\
\hdashline \gamma_{2}^{\prime \prime}
\end{array}\right]+\left[\begin{array}{c:c}
A_{11} & \overline{0} \\
A_{21} & \overline{0} \\
\hdashline-B_{11} & \overline{0} \\
\hdashline \overline{0} & -B_{22}^{-1}
\end{array}\right]\left[\begin{array}{c}
\overline{\gamma_{1}^{\prime \prime}} \\
\overline{\mathrm{v}_{2}^{\prime \prime}}
\end{array}\right]=\overline{0}
$$

$k=1$;

$\left[\begin{array}{c:c}E & A_{22} \\ \hdashline 0 & E\end{array}\right]\left[\begin{array}{l}\overline{\gamma_{k}^{\prime}} \\ \hdashline \gamma_{k}^{\prime \prime}\end{array}\right]+\left[\begin{array}{c}\overline{0} \\ \hdashline-B_{22}^{-1}\end{array}\right]\left[\overline{\mathrm{v}_{k}^{\prime \prime}}\right]=\overline{0}, \quad k=\overline{2, M}$.

When inserting dependent variables $\left(\Delta \delta, \Delta Q_{1}, \Delta V_{2}\right.$ and $\boldsymbol{\gamma}^{\prime}, \boldsymbol{v}_{\mathbf{1}}^{\prime \prime}, \boldsymbol{\gamma}_{\mathbf{2}}^{\prime \prime}$ ) into (17), (20) through independent variables $\left(\Delta V_{1}, \Delta Q_{2}\right.$ and $\left.\boldsymbol{\gamma}_{\mathbf{1}}^{\prime \prime}, \boldsymbol{v}_{\mathbf{2}}^{\prime \prime}\right)$ considering objective function $F$ (11) and component (12) upon transition to infinitesimal increments of the variables, the expression of reduced gradient simulating loads only with the first actively constrained GLD (13), will be:

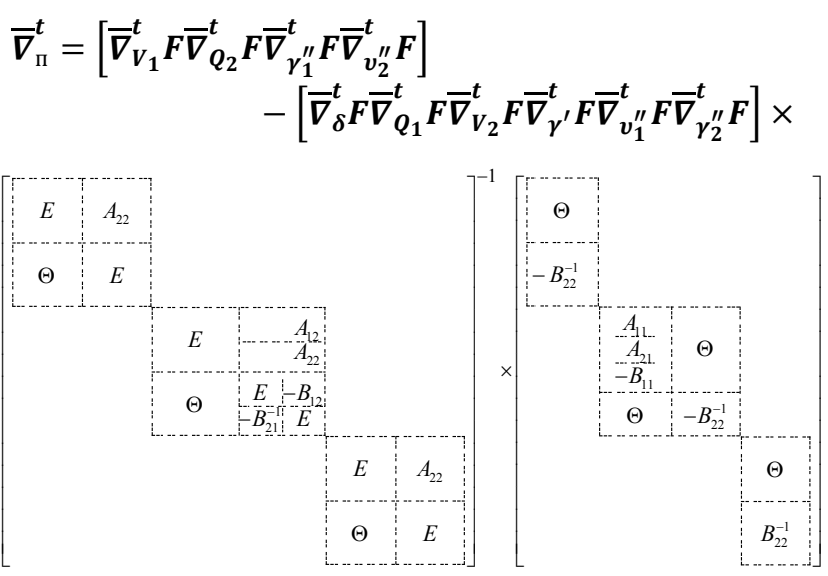

where $\bar{\nabla}_{\Pi}^{t}$ is a $G(1+M)$-dimensional reduced gradient vector with components; $\overline{\boldsymbol{\nabla}}_{\boldsymbol{V}_{\mathbf{1}}}^{\boldsymbol{t}} \boldsymbol{F} \overline{\boldsymbol{\nabla}}_{\boldsymbol{Q}_{2}}^{\boldsymbol{t}} \boldsymbol{F}$ and $\overline{\boldsymbol{\nabla}}_{\gamma_{1}^{\prime \prime}}^{\boldsymbol{t}} \boldsymbol{F} \overline{\boldsymbol{\nabla}}_{\boldsymbol{v}_{2}^{\prime \prime}}^{\boldsymbol{t}} \boldsymbol{F}$ are vector-rows $\left[\boldsymbol{\partial F} / \boldsymbol{\partial Y}\right.$ ], each being of total size $G ; \overline{\boldsymbol{\nabla}}_{\boldsymbol{\delta}}^{\boldsymbol{t}} \boldsymbol{F}$ and $\overline{\boldsymbol{\nabla}}_{\gamma^{\prime}}^{\boldsymbol{t}} \boldsymbol{F}$ are $N$-dimensional vector-rows $[\boldsymbol{\partial \boldsymbol { F }} / \boldsymbol{\partial X}]$;

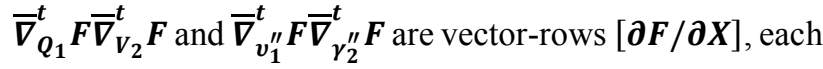
of total size $N$. In expression (22), $2 N(1+M)$-dimensional square matrix $[\boldsymbol{\partial W} / \boldsymbol{\partial X}]^{-1}$ and $2 N(1+M) \times G(1+M)$ dimensional matrix $[\partial W / \partial Y]$ are used.

The modified model based on GRG method (22) allows stochastic optimization of the objective function $F$ in the space of expectations of the modes parameters, eigenvectors of wattage MCM and modeling coefficients $F=f\left(Q_{i}, \delta_{i}, V_{i}, v_{k i}^{\prime \prime}, \gamma_{k i}^{\prime}, \gamma_{k i}^{\prime \prime}\right)$, taking into account the ES multimoding in a concise form.

\section{Multimode-based optimal choice of loading RP sources}

An objective function of the total EE losses (11), (12) is determined by the mathematical expectations of the mode parameters, the components of the eigenvectors and 
modeling coefficients: $F=f\left(Q_{i}, \delta_{i}, V_{i}, \cup_{k i}^{\prime \prime}, \gamma_{k i}^{\prime}, \gamma_{k i}^{\prime \prime}\right)$. It can be minimized by means of modifying GRG method (13) - (22) based on the stochastic model of loads and the set of modes (1), (2), (6) - (10) specifying the active resistance of overhead and cable lines in (11) by the average values of current loads and ambient temperatures for the period under consideration. An optimization step is calculated as a minimum of the values determined from the condition for observing the constraints in the form of simple inequalities for the mode parameters $\mathrm{O}$ and $\mathrm{V}$ and by the parabolic interpolation method, provided that the EE loss function passes through its minimum inside the constraints. The mode dependent parameters enter the possible domain of mathematical expectations (obtaining a possible point of the optimization trajectory) by means of solving the SSM nonlinear equations through Newton's method. Corrections of dependent variables for average loads are determined from solving the systems of (17), (18), and variables $\gamma^{\prime}, U_{1}^{\prime \prime}, \gamma_{2}^{\prime \prime}$, which model the deviations of the optimized variables from the average can be found from the solution of linear systems (20), (21).

The variables found during the optimization allow obtaining:

1. A criterion (objective) function of total EE losses and its components (the value of EE load losses (11) and idle running (12) in the initial and optimal states).

2. Ranges of alteration of the optimized mode parameters (14) taking into account expressions (9) and (10) for variances.

3. Diagrams (curves) of loading of the RP sources (2) and voltages (6) in the system nodes in the given time interval.

\section{The main stages of the algorithm for optimal compensation of reactive loads are as follows}

The initial data in the optimization problem are simulated (or initial) curves of the active and reactive powers of the nodes (2), presented through average loads using GLD (1). Reactive powers for $G$ set of RP sources (including compensating devices) are the main independent variables, determined during the solution process and written in the same form in which the initial diagrams are given (2):

$$
Q_{l j}=M Q_{l}+\sum_{k=1}^{K} v_{k l}^{\prime \prime} \Gamma_{k j}, \quad l=\overline{1, G}, j=\overline{1, d} .
$$

Expressions (23) differ from similar expressions (2) in the fact that the diagrams (curves) $Q_{l j}$ of RP generation and, accordingly, expectations $M Q_{l}$ and coefficients $\mathbf{v}_{\boldsymbol{k} \boldsymbol{l}}$ are not specified, but they are determined in the process of solving the optimization problem.

The greatest optimal power of compensation at node $i$ of $G$ taking into account (14) shall be

$$
Q_{i}^{\mathrm{nc}}=M Q_{i}+k_{\beta}^{\max } \sigma Q_{i} .
$$

GRG-based algorithm for optimal compensation of reactive loads starts to work and performs each subsequent optimization step from a possible point $\bar{Z}=(\bar{Y}, \bar{X})$ in accordance with the following steps:

1. Determines a possible vector of the parameters of the basic electric mode corresponding to the load expectations for the initial (starting) point of the optimization search.

2. Computes the objective function and a number of derivatives of the objective function and imbalance functions of $\partial \Phi / \partial Z, \partial \Phi / \partial \gamma, \partial \mathrm{W} / \partial \mathrm{Z}$ to model the constraints and form the expression of the reduced gradient and other calculated expressions.

3. Determines the vectors: of the reduced gradient $\bar{\nabla}_{\mathrm{r}}$, the permissible directions of the optimization descent $\bar{\Delta}=\left(\bar{\Delta}_{Y}, \bar{\Delta}_{X}\right)$ with respect to the independent and dependent variables, and the step size $\lambda$ of external iterations in the selected optimization direction.

4. Calculates a new vector of the variables as $\widetilde{Z}^{(k+1)}=\left(\bar{Y}^{(k)}+\lambda \bar{\Delta}_{Y}^{(k+1)}, \bar{X}^{(k)}+\lambda \bar{\Delta}_{X}^{(k+1)}\right)$ at $(k+1)$ external step, which in general is infeasible since it is determined by linear translation along $\lambda \bar{\Delta}$ vector relatively to nonlinear constraints of the form of SSM equations.

5. Adjusts the dependent parameters $\bar{V}, \bar{\delta}, \bar{\gamma}$ to obtain a valid vector of variables $\bar{Z}^{(k+1)}$. The main part of this procedure is the solution of the equations of balance constraints as in (17) for fixed values of the RP sources and the further analysis of the parameters of the basic steadystate mode with subsequent verification of interval constraints (13). If the resulting voltages do not satisfy the controlled constraints (13), it is necessary to obtain new values of the controlled variables (RP sources) by decreasing $\lambda$ step or by means of fixing the violated limits on the limit values (change of basis), and then redetermine the dependent variables.

6. Controls of the decline of the objective function at $(k+1)$ iterative step and the fulfillment of the criteria for the termination of the optimization search.

The calculation cycles for 2-6 are iterated until the optimum condition is satisfied i.e. the minimum of the objective function of the total EE losses.

Software for optimization algorithms. These algorithms form the basis of ORESA stochastic optimization software [10] based on algorithms and OPRES instant mode optimization software [11]. ORESA is intended for optimal distributing of reactive loads of existing RP sources over a time interval according to the criterion of minimum EE losses and aims to solve the problems of ES optimal functioning in various mode planning cycles.

\section{Conclusions}

We implemented the proposed modification of the generalized reduced gradient method for stochastic 
modeling of multimode-based electrical systems. The program provides ranges and diagrams of loadings for RP and voltages changes of RP sources and other ES nodes, $\mathrm{EE}$ losses in the initial and optimal states avoiding analyzing and optimizing of the modes at each load stationarity interval. The accuracy of ORESA was assessed via statistical tests as a result of direct reproduction of the totality of typical optimal modes on a variety of circuits of 35-220 kV electrical grids and systems: the accuracy achieved for solving this particular operational problem was sufficient for actual practices.

\section{References}

1. Belyaev L.S. Primenimost veroyatnostnykh metodov $v$ energeticheskikh raschetakh [Applicability of Probabilistic Methods in Energy Calculations], Belyaev L.S., Krumm L.A. Izvestiya Akademii nauk [Proceedings of the Academy of Sciences]. Energetika i transport [Energy and Transport], 1983 No. 2. pp. 3 -11 (in Russian).

2. I. V. Shulgin. Modified Stochastic Estimation of Load Dependent Energy Losses in Electric Distribution Network, I. V. Shulgin, A. A. Gerasimenko, Su Quan Zhou. International Journal of Electrical Power \& Energy Systems, Vol. 43, Issue 1, December 2012, pp. 325-332 (JEPE1673, www.sciencedirect.com/science/article/pii/S0142061 512000890) (indexed in Web of Science u Scopus).

3. Gerasimenko A.A. Stokhasticheskii metod rascheta nagruzochnykh poter elektroenergii $v$ raspredelitelnykh elektricheskikh setyakh [Stochastic Method for Calculating Energy Load Losses in Electric Distribution Network], Gerasimenko A.A., Shulgin I.V. Moscow. Elektricheskie stantsii [Electric Power Stations], 2013, No. 4. pp. 44-59 (in Russian).

4. Himmelblau D. Prikladnoe nelineinoe programmirovanie [Applied Nonlinear Programming] / Himmelblau D. Moscow: Mir Publishing House, 1975. 534 p. (in Russian).
5. Krumm L.A. Metody privedennogo gradienta pri upravlenii elektroenergeticheskimi sistemami [Reduced Gradient Methods for Controlling Electric Power Systems], Krumm L.A. Novosibirsk, Nauka Publishing House, 1977. 368 p. (in Russian).

6. Lowly D. Faktornyi analiz kak statisticheskii metod [Factor Analysis as a Statistical Method]/ Lowly D., Maxwell A. Moscow, Mir Publishing House, 1967. 144 p. (in Russian).

7. Harmann G. Sovremennyi faktornyi analiz [Modern Factor Analysis]/ Harmann G., Moscow: Statistika [Statystics], 1972. 486 p. (in Russian).

8. Gerasimenko A.A. Optimizatsiya rezhimov elektricheskikh sistem na osnove metoda privedennogo gradienta [Optimization of the Modes of Electrical Systems based on the Reduced Gradient Method], Gerasimenko A.A., Lipes A.V., Moscow, Elektrichestvo [Electricity], 1989. No. 9. pp. 1- 7. (in Russian).

9. Gerasimenko A.A. Vybor kompensiruyushchikh ustroistv $v$ raspredelitelnykh setyakh elektroenergeticheskikh sistem [Compensating Devices in Distribution Networks of Electric Power Systems], Gerasimenko A.A., Neshataev V.B., Moscow. Elektrichestvo [Electricity], 2014. No. 4. pp. $4-17$. (in Russian).

10. Gerasimenko A.A. Programma optimizatsii rezhimov elektroenergeticheskikh sistem po reaktivnoi moshchnosti na intervale vremeni "ORESA" [ORESA Optimization Software for the Modes of Electric Power Systems by Reactive Power on the Time Interval], Gerasimenko A.A., Neshataev V.B., Rossiiskaya Federatsiya [Russian Federation], No. 2012610439 as of 10 January 2012 (in Russian).

11. Gerasimenko A.A. Programma optimizatsii rezhimov elektroenergeticheskikh sistem po reaktivnoi moshchnosti "OPRES" [OPRES Reactive Power Mode Optimization Software], Gerasimenko A.A., Neshataev V.B. Rossiiskaya Federatsiya [Russian Federation] No. 2012610438 as of 10 January 2012 (in Russian). 